\title{
Diseño de estudios epidemiológicos
}

\author{
Mauricio Hernández-A vila, Ph.D., ${ }^{(1)}$ Francisco Garrido-Latorre, M. en C., ${ }^{(2)}$ \\ Sergio López-Moreno, M.C. ${ }^{(2)}$
}

L os principales objetivos de la investigación epidemiológica son, por un lado, describir la distribución de las enfermedades y eventos de salud en poblaciones humanas y, por otro, contribuir al descubrimiento y caracterización de las leyes que gobiernan o influyen en estas condiciones. La epidemiología no representa un dominio del conocimiento claramente delimitado como el que tienen otras ciencias médicas como, por ejemplo, la bioquímica o la fisiología. La epidemiología se emplea en las distintas ramas de la medicina como una herramienta para el estudio de diferentes enfermedades o eventos relacionados con la salud, especialmente cuando se busca evaluar la repercusión de éstos en el ámbito de la población. Así, es posible encontrar aplicaciones de la epidemiología tanto para definir los mecanismos de transmisión de una enfermedad infecciosa como para evaluar la respuesta médica organizada para contender con la misma o para evaluar el impacto, en el ámbito poblacional, del desarrollo de resistencia a los diferentes tratamientos. El principal objetivo de la epidemiología es desarrollar conocimiento de aplicación a nivel poblacional (cuadro I), y por esta razón es considerada como una de las ciencias básicas de la salud pública.

La información necesaria para cumplir con los objetivos de la investigación epidemiológica, ya sea de tipo descriptivo o analítico, se deriva de la experimentación con seres humanos o, más frecuentemente, de la observación directa de grupos poblacionales. A pesar de que para la epidemiología es de interés princi- pal derivar conocimiento de aplicación poblacional, raramente estudia a la población en su conjunto. Por ello, tanto para la experimentación con voluntarios como para la observación de grupos poblacionales es necesario desarrollar estrategias muestrales y de medición que permitan, en primera instancia, estudiar subgrupos de la población y, en un segundo término, hacer extrapolaciones del conocimiento generado hacia el total de la población. La validez de la información derivada de los estudios epidemiológicos depende de manera importante de lo adecuado y apropiado de los métodos utilizados. El reconocimiento de la importancia que tienen los aspectos metodológicos como un

\section{Cuadro I \\ Principales usos de la epidemiología en SALUD PÚBLICA}

- Identificación de la historia natural de las enfermedades

- Descripción de la distribución, frecuencia y tendencias de la enfermedad en las poblaciones

- Identificación de la etiología y los factores de riesgo para la aparición y desarrollo de enfermedades

- Identificación y explicación de los mecanismos de transmisión y diseminación de las enfermedades

- Identificación de la magnitud y tendencias de las necesidades de salud

- Identificación de la magnitud, vulnerabilidad y formas de control de los problemas de salud

- Evaluación de la eficacia y efectividad de las intervenciones terapéuticas

- Evaluación de la eficacia y efectividad de la tecnología médica

- Evaluación del diseño y ejecución de los programas y servicios de salud

(1) Centro de Investigación en Salud Poblacional, Instituto N acional de Salud Pública (IN SP), México.

(2) Centro de Investigación en Sistemas de Salud, IN SP, México.

Solicitud de sobretiros: Mauricio Hernández Avila, Centro de Investigación en Salud Poblacional, Instituto N acional de Salud Pública. A venida Universidad 655, colonia Santa María A huacatitlán, 62508 Cuernavaca, Morelos, México.

Correo electrónico: mhernan@insp3.insp.mx 
eje necesario para el desarrollo y avance del conocimiento epidemiológico ha propiciado que se asuma como un objetivo mismo de la epidemiología el desarrollo y el estudio de nuevos métodos de aplicación en el campo. Esto, sin duda, ha contribuido de manera importante a mejorar la calidad y la validez del conocimiento derivado de estudios epidemiológicos y a consolidar a la epidemiología como una ciencia básica necesaria para el avance de la salud pública y de la medicina.

En este trabajo se hace una revisión y una actualización de los principales diseños epidemiológicos utilizados en investigaciones de tipo epidemiológico para conformar los grupos poblacionales de estudio. Asimismo, se propone un esquema de clasificación de dichas estrategias, conforme a una escala ordinal en términos de la evidencia que aporta cada diseño para establecer relaciones causa efecto entre dos variables de interés.

En diferentes libros de texto y trabajos que abordan la aplicación y desarrollo de los métodos epidemiológicos se han propuesto diversos esquemas para agrupar y caracterizar a los distintos tipos de estudio, los cuales se han clasificado de acuerdo con: a) el tipo de asignación de la exposición o variable en estudio; b) el número de mediciones que se realiza en cada sujeto de estudio para verificar la ocurrencia del evento o cambios en la exposición; c) la temporalidad del inicio de la exposición o de la ocurrencia del evento; d) los criterios utilizados para la selección de la población a estudiar, y e) la unidad de análisis donde se mide el evento en estudio (cuadro II). ${ }^{1-5}$
En términos de causalidad la asignación de la exposición ${ }^{*}$ es el criterio más importante de clasificación y divide a los estudios epidemiológicos en tres tipos: a) experimentales, cuando el investigador controla la exposición y utiliza la aleatorización como método de asignación; b) pseudo-experimentales (o de intervención no aleatorizados), cuando el investigador controla la exposición pero no utiliza procedimientos de aleatorización en la asignación, y c) no-experimentales u observacionales, cuando la exposición ocurre sin la participación del investigador y de acuerdo con variables que están fuera de control del investigador.

De acuerdo con el número de mediciones que se realiza en cada sujeto de estudio para medir la ocurrencia del evento $\mathrm{o}$ cambios en la variable de exposición a lo largo del tiempo, los estudios se pueden dividir en: a) longitudinales, cuando se realizan al menos dos mediciones: la medición basal para determinar el estado inicial y una subsecuente para

\footnotetext{
* En este trabajo utilizaremos el término exposición para referirnos a la variable en estudio. Utilizaremos exposición como un término de significado amplio, que puede abarcar desde la exposición a una bacteria o una sustancia tóxica hasta la exposición a un suplemento nutricional, una vacuna, un programa de salud o un estilo de vida.

‡ Utilizaremos el término evento para referirnos a la variable respuesta o cambio que se espera detectar con relación a la exposición. Se utiliza también de manera amplia y puede referirse tanto a la ocurrencia de una enfermedad, como al cambio de estado o cambio promedio en una variable continua; por ejemplo, cambio en las concentraciones de colesterol sérico o seroconversión después de aplicada una vacuna.
}

\section{Cuadro II}

Clasificación de los estudios epidemiológicos

\begin{tabular}{|c|c|c|c|c|c|}
\hline Tipo de estudio & Asignación de la exposición & $\begin{array}{l}\text { Número de } \\
\text { observaciones } \\
\text { por individuo }\end{array}$ & $\begin{array}{l}\text { Criterios de selección } \\
\text { de la población } \\
\text { en estudio }\end{array}$ & Temporalidad & $\begin{array}{c}\text { Unidad } \\
\text { de análisis }\end{array}$ \\
\hline Ensayo aleatorizado & Aleatoria & Longitudinal & $\mathrm{N}$ inguno & Prospectivo & Individuo \\
\hline Pseudo-experimentales & Por conveniencia & Longitudinal & $N$ inguno & Prospectivo & Individuo \\
\hline Cohorte & Fuera de control del investigador & Longitudinal & Exposición & $\begin{array}{l}\text { Prospectivo } \\
\text { o retrospectivo }\end{array}$ & Individuo \\
\hline Casos y controles & Fuera de control del investigador & Longitudinal o transversal & Evento & $\begin{array}{l}\text { Prospectivo } \\
\text { o retrospectivo }\end{array}$ & Individuo \\
\hline Estudio de encuesta & Fuera de control del investigador & Transversal & $N$ inguno & Retrospectivo & Individuo \\
\hline Ecológico o de conglomerado & Fuera de control del investigador & Longitudinal o transversal & Ninguno & Retrospectivo & $\begin{array}{l}\text { Grupo } \\
\text { (o población }\end{array}$ \\
\hline
\end{tabular}


determinar la ocurrencia del evento, y b) transversales, cuando se realiza una sola determinación en los sujetos de estudio y se evalúan de manera concurrente la exposición y el evento de interés.

En términos de causalidad existe una diferencia importante entre estos dos tipos de estudio, ya que en los longitudinales es posible verificar que la exposición antecede a la ocurrencia del evento, con lo que se cumple el principio temporal de causalidad -la causa antecede al efecto; en tanto que en los transversales resulta imposible verificar este tipo de relaciones cuando se estudian exposiciones que cambian con el tiempo. Los estudios transversales sí pueden proporcionar información valiosa cuando se estudian factores que no varían (como el sexo y la carga genética) o exposiciones únicas que no cambian con el tiempo (por ejemplo, el caso de la población expuesta a una bomba atómica).

El criterio de temporalidad en la ocurrencia del evento se utiliza para distinguir entre los estudios retrospectivos y prospectivos. El punto de referencia para esta clasificación es la ocurrencia del evento de interés (la variable respuesta). Si al inicio del estudio, el evento investigado ya ocurrió y el investigador planea reconstruir su ocurrencia en el pasado utilizando registros o entrevistando a los mismos sujetos de estudio, se considera que el estudio es retrospectivo. Si la ocurrencia del evento se registra durante el estudio, es decir, si los sujetos de estudio están libres del evento de interés al iniciar su participación en el estudio, el diseño se considera de tipo prospectivo. En general, podríamos afirmar que los estudios prospectivos tienen mayor puntaje en la escala de causalidad, dado que en este tipo de estudios se pueden diseñar instrumentos para la medición y registro del evento que aseguren la calidad de las mediciones. En cambio, en los estudios retrospectivos la calidad de medición y registro del evento dependen con frecuencia de instrumentos que no fueron diseñados de manera expresa para observar el evento en cuestión ni para responder a los objetivos de la investigación. Los estudios que incluyen eventos que ocurrieron antes de iniciar la investigación y eventos evaluados de manera prospectiva son referidos en algunos textos como mixtos o ambispectivos. $^{3}$

La selección de los participantes en el estudio se puede llevar a cabo de acuerdo con la exposición, el evento o sin considerar ninguna de estas características de los sujetos elegibles para el estudio. La selección con base en estos atributos se utiliza con frecuencia para distinguir entre los diferentes estudios epidemiológicos de tipo observacional. Cuando los sujetos son seleccionados con base en la exposición, es decir, se elige un grupo expuesto y uno no expuesto, en los que posteriormente se determinará la ocurrencia del evento, se considera que se trata de un estudio de cohorte. En contraste, cuando se selecciona a los participantes con base en el evento de estudio, es decir, se elige de manera independiente un grupo de sujetos que tienen el evento de interés (casos) y un grupo de sujetos que no lo tienen (controles) y en estos grupos se determina la exposición, entonces hablamos de un estudio de casos y controles. Finalmente, cuando la selección es indistinta de la ocurrencia de la exposición o del evento, es decir, los sujetos de estudio son seleccionados sin considerar información sobre la exposición o el evento, y la ocurrencia de éstos se determina una vez conformada la población en estudio, entonces los estudios se denominan de encuesta, aunque algunos textos también los clasifican como de tipo transversal. ${ }^{2}$ La característica principal de esta última estrategia de muestreo es que la evaluación de la exposición y de la ocurrencia del evento se hacen de manera simultánea. En términos de causalidad se puede decir, de manera general, que los estudios de cohorte tienen mayor peso que los estudios de casos y controles y que los transversales. Sin embargo, es importante mencionar que los estudios de casos y controles, con ciertas características, pueden ser tan informativos (en términos de causalidad) como un estudio de cohorte.

Por último, la unidad de análisis se ha utilizado para clasificar a los estudios en ecológicos (de conglomerados) e individuales. A diferencia de los estudios individuales, en los que la unidad de análisis es el individuo y se cuenta con al menos una medición de cada uno de ellos, en los estudios ecológicos la unidad de análisis es un grupo (por ejemplo, un país o una región) y se cuenta con el promedio de eventos o de exposición para el grupo, desconociéndose a nivel individual la condición de evento o exposición para cada individuo de la población. Este tipo de estudios conlleva problemas importantes en su interpretación ya que, dado que los datos se encuentran agrupados, no es posible corregir por diferencias en otras variables (posibles variables confusoras*) que pudieran explicar los resultados observados. Por esta razón, los resultados de este tipo de estudios tienen el peso más bajo en la escala de causalidad.

A continuación describiremos brevemente las características de las principales estrategias epidemiológicas utilizadas para estudiar grupos poblacionales.

\footnotetext{
* Una variable confusora está asociada con la exposición y el evento en estudio, por lo que una diferencia en su distribución en los grupos que se comparan puede distorsionar los resultados.
} 


\section{Ensayos epidemiológicos aleatorizados}

Los ensayos epidemiológicos aleatorizados son estudios experimentales que, cuando se llevan a cabo de manera adecuada, proporcionan el máximo grado de evidencia para confirmar la relación causa efecto entre la exposición y el evento en estudio. Se distinguen de los estudios observacionales (no experimentales) porque el investigador tiene control sobre la asignación de la exposición y porque ésta se lleva a cabo mediante un proceso aleatorio. Además, dado que se trata de estudios longitudinales y prospectivos, y en los que la unidad de análisis es el individuo, es posible prevenir la introducción de sesgos y lograr altos índices de validez. En este tipo de estudios es posible minimizar la ocurrencia de sesgos mediante tres procedimientos, los cuales tienen como objetivo garantizar la comparabilidad: a) de intervenciones (o de la exposición), b) de los grupos en estudio, y c) de los procedimientos para recopilar la información obtenida de la población en estudio. ${ }^{5}$

La comparabilidad de intervenciones es indicativa de la "pureza" del contraste entre los grupos experimentales, y se refiere a que la única diferencia entre las exposiciones que se comparan es la parte o sustancia activa de la exposición en estudio. El concepto de comparabilidad de intervenciones se puede entender como una extensión en epidemiología del concepto de efecto placebo observado de los ensayos clínicos. Este concepto se deriva del hecho de que el efecto de un medicamento es el resultado de la suma de dos componentes: uno, causado por la sustancia activa del medicamento y, otro, producido por la acción de recibir atención o por el componente psicológico asociado con la idea de recibir un medicamento. La idea de identificar una intervención exactamente igual a la que se pretende probar, pero sin la sustancia activa, es precisamente eliminar de la comparación el efecto atribuido al placebo y de esta manera estimar únicamente la diferencia atribuible a la sustancia activa o intervención en cuestión.

La comparabilidad de poblaciones se logra cuando los grupos experimentales que reciben las diferentes intervenciones son similares en todas y cada una de las características que pudieran tener relación con el evento en estudio o con la manera en que actúa la exposición. En términos epidemiológicos este concepto indica la ausencia de factores de confusión o modificación de efecto. En una situación ideal, la comparabilidad de poblaciones se podría lograr observando a los mismos sujetos en estudio en condiciones experimentales diferentes. Sin embargo, las condiciones necesarias para que esto ocurra raramente se consiguen en el contexto epidemiológico. Como alternativa para lograr la comparabilidad de poblaciones se ha utilizado la aleatorización. Mediante este proceso se deja al azar la distribución de los sujetos en los diferentes grupos experimentales y se espera que en promedio los grupos tengan características comparables. Es importante mencionar que la comparabilidad que se obtiene con la asignación aleatoria a los grupos experimentales depende del tamaño muestral, y que la simple aleatorización no garantiza completamente que las variables se distribuirán homogéneamente en los distintos grupos de intervención. Dado que las variables se distribuyen al azar será siempre necesario verificar el resultado de la aleatorización, debido a que existe la posibilidad de no funcionar adecuadamente. Esto se puede llevar a cabo comparando la distribución de las variables medibles en los diferentes grupos experimentales. La distribución homogénea de estas variables entre los grupos experimentales sería indicativa de éxito en la aleatorización.

Por último, la comparabilidad de la información se logra cuando se utilizan exactamente los mismos métodos de seguimiento y de medición en todos los participantes en el estudio. Una manera de lograr esto es cegando o enmascarando a los evaluadores y a los participantes en el estudio respecto a la condición de exposición. Si el o los evaluadores desconocen la pertenencia de los sujetos que evalúan, con relación a los grupos experimentales, es probable que la medición no se vea afectada por esta información. La "ignorancia", en este caso, hace que los grupos sean tratados en igual forma. Si se pusiera especial interés en determinar la ocurrencia del evento en el grupo experimental, los resultados podrían ser erróneos dada la diferencia en los procedimientos utilizados. Este concepto abarca tanto la calidad de la información como la proporción de los sujetos en estudio que se pueden perder durante el seguimiento en cada grupo experimental. La pérdida diferencial de participantes puede ocasionar errores importantes. Algunos textos refieren este último problema dentro de los sesgos de selección. ${ }^{2}$

Los pasos para la realización de este tipo de estudios (figura 1) incluyen la definición de la población blanco, que es aquella a la cual se pretenden extrapolar los resultados del estudio. Al aplicar los criterios de inclusión en el estudio se define la población elegible y de esta población se seleccionan los participantes en el estudio; esto último se puede llevar a cabo ya sea mediante el reclutamiento de voluntarios o mediante la selección de una muestra representativa de la población blanco. Es importante mencionar que siempre que se trabaja con poblaciones humanas se tendrá un grupo 


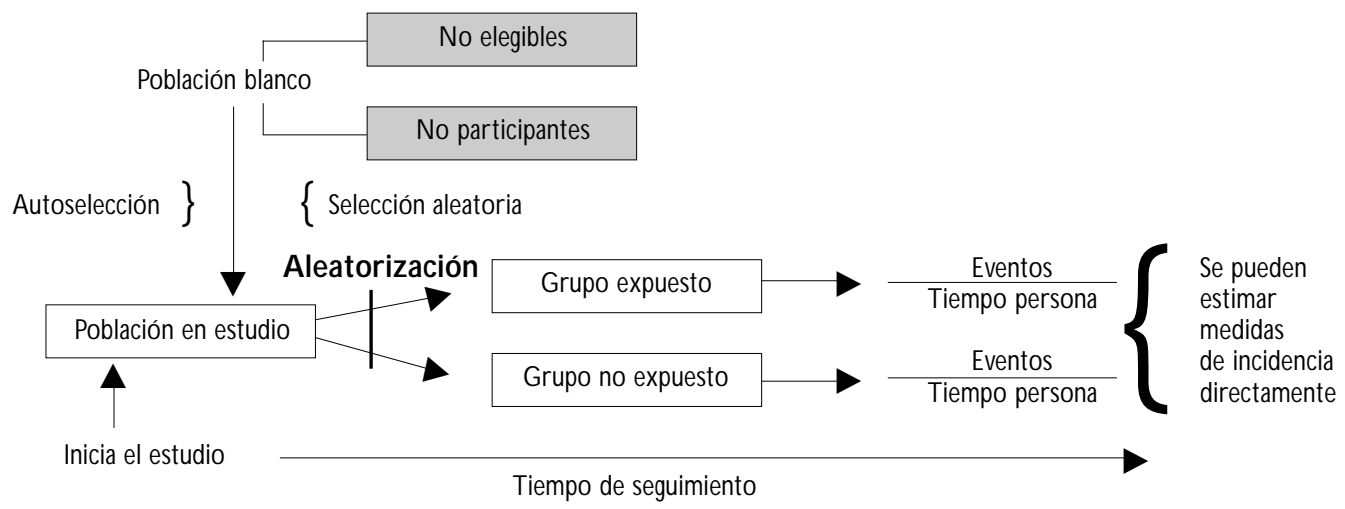

\section{Figura 1. Ensayo clínico aleatorizado}

autoseleccionado de la población que corresponde a aquellos sujetos que otorgan el consentimiento informado para participar en el proceso experimental. La autoselección o la selección de un grupo representativo de la población blanco tiene un alto grado de importancia en términos de la validez externa* de los resultados, ya que éstos serán aplicables a la población blanco en la medida de que la población en estudio represente adecuadamente a esta población. Una vez identificados los participantes en el estudio y que éstos han dado su consentimiento para participar en el proceso experimental, los sujetos se asignan de manera aleatoria a los grupos experimentales (figura 1). Posteriormente, los sujetos se siguen en el tiempo con el fin de documentar la ocurrencia del evento en estudio y posibles cambios en otras covariables de interés.

Algunos textos ${ }^{4}$ mencionan la existencia de un grupo intermedio entre los estudios experimentales y no experimentales; éstos son llamados quasi-experimentales. En estos diseños el investigador controla la asignación de la exposición, sin embargo, esta asignación no se hace de manera aleatoria. Este tipo de estudios de intervención incluye los diseños tipo antes $y$ después o de comparación concurrente.

En los estudios observacionales (no experimentales) la asignación de la exposición ocurre sin la participación del investigador. En este tipo de diseños es común que la exposición ya haya ocurrido al iniciar el estudio, y que ésta se haya dado por algún factor independiente y fuera del procedimiento experimental.

\footnotetext{
* Validez externa: se refiere a la generalización de los resultados
} observados.

\section{Estudios de cohorte}

Entre los estudios observacionales, este tipo de diseño representa lo más cercano al diseño experimental y también tiene un alto valor en la escala de causalidad, ya que es posible verificar la relación causa efecto correctamente en el tiempo. Sin embargo, dado que se trata de estudios observacionales tienen la importante limitación de que la asignación de la exposición no es controlada por el investigador ni asignada de manera aleatoria, por lo que no es posible controlar completamente las posibles diferencias entre los grupos expuesto y no expuesto en relación con otros factores asociados con la ocurrencia del evento.

La selección de los participantes con base en la exposición caracteriza a los estudios de cohorte (figura 2). En este tipo de diseño epidemiológico la población en estudio se define a partir de la exposición y debe estar conformada por individuos en riesgo de desarrollar el evento en estudio. Los sujetos de estudio se seleccionan de la población que tiene la exposición de interés y de grupos poblacionales comparables, pero que no tienen la exposición. Una vez conformada la población en estudio ésta se sigue en el tiempo y se registra en ella la ocurrencia del evento de interés o variable respuesta.

El diseño de cohorte es especialmente eficiente para estudiar exposiciones raras o poco frecuentes; por ejemplo, las exposiciones ocupacionales que se presentan en poblaciones muy reducidas de trabajadores. En general, cuando se requiere evaluar los riesgos asociados con algún tipo particular de ocupación, se selecciona a grupos ocupacionales y se establece un grupo de comparación (no expuesto) tomado de la población general o, incluso, ubicado en la misma in- 
dustria o en otra similar, pero no en contacto con la exposición en estudio.

Los estudios de cohorte también se utilizan regularmente para estudiar exposiciones que se presentan con una alta frecuencia en la población general. Para este tipo de exposiciones es común seleccionar aleatoriamente grupos representativos de la población que posteriormente se clasifican de acuerdo con la exposición; la cohorte (población en estudio) queda conformada con los participantes que no tienen el evento en estudio y que están en riesgo de desarrollar el evento, posteriormente este grupo se sigue en el tiempo con el fin de registrar la ocurrencia del evento. El procedimiento antes descrito se refiere a un estudio prospectivo (figura 2); sin embargo, los estudios de cohorte también pueden ser retrospectivos (figura 3). En este tipo de estudios, se inicia con la definición de los grupos expuesto y no expuesto en algún punto en el pasado y posteriormente se reconstruye la experiencia de la cohorte en el tiempo, identificando a los sujetos en el tiempo actual (cuando se realiza el estudio) y evaluando si a la fecha referida ya han desarrollado el evento de interés.

En su concepción más simple (figuras 2 y 3), un estudio de cohorte consiste en seleccionar un grupo expuesto y otro no expuesto de la población elegible, observarlos durante un tiempo determinado y compararlos en términos de la ocurrencia del evento de interés. La validez de la comparación dependerá de que no existan diferencias (aparte de la exposición) entre los grupos expuesto y no expuesto. Cualquier diferencia con relación a una tercera variable entre el grupo expuesto y no expuesto que esté relacionada con la ocu-

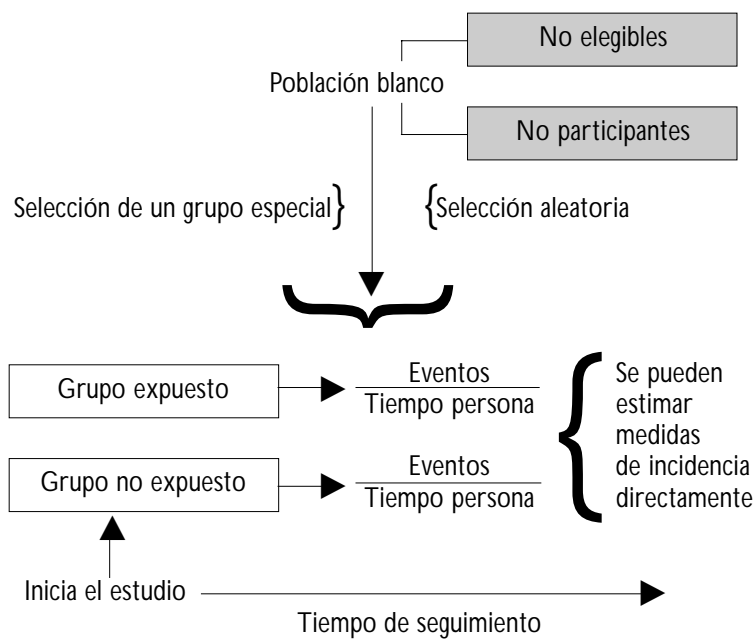

Figura 2. Estudios de cohorte prospectiva rrencia del evento podría distorsionar los resultados sobre la asociación real entre la exposición y el evento.

Los estudios de cohorte son difíciles de realizar y, además, son costosos. Se considera que este tipo de diseño es poco eficiente para el estudio de enfermedades raras, ya que para registrar un número adecuado de eventos se requeriría un número muy grande de participantes y de tiempos prolongados de seguimiento (cuadro III).

\section{Estudios de casos y controles}

Durante los últimos años se ha desarrollado una estrategia de selección sobre la base del evento en estudio,

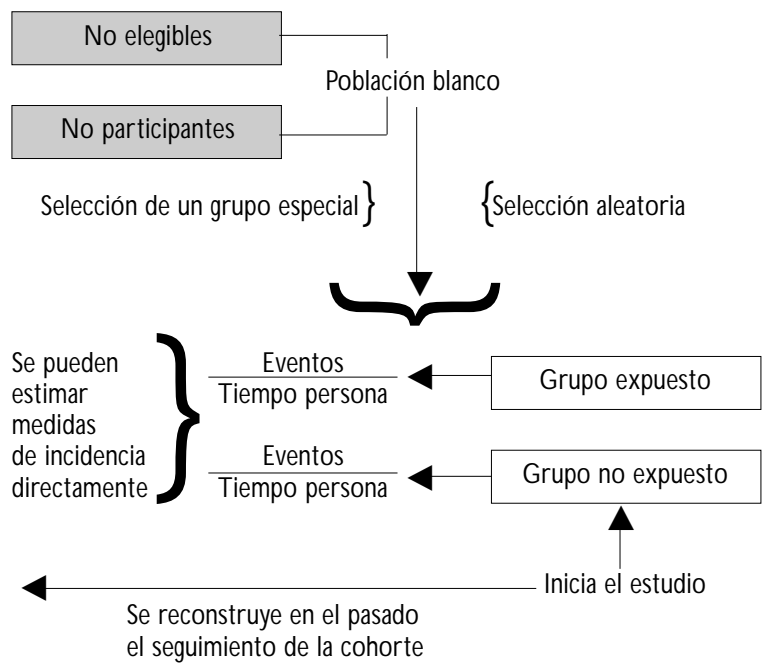

Figura 3. Estudios de COHORTE RETROSPECTIVA

\section{Ventajas y DesVentajAS DE LOS ESTUdios DE COHORTE}

Ventajas

- Más cercanos a un experimento

- La relación temporal causa efecto es verificable

- Se pueden estimar medidas de incidencia

- Eficientes para evaluar exposiciones poco frecuentes

- Se pueden estudiar varios eventos

- Se pueden fijar criterios de calidad en la medición del evento

- Bajo riesgo de sesgo de selección (en especial en estudios prospectivos)

Desventajas

- Cuando se trata de eventos poco frecuentes la complejidad y el costo pueden aumentar considerablemente, ya que requiere estudiar y seguir un número grande de participantes

- Son estudios difíciles de realizar 
este tipo de muestreo se ha denominado en la literatura epidemiológica como estudios de casos y controles, ${ }^{1-4}$ o casos y referentes. ${ }^{5}$ La característica principal de este diseño epidemiológico es que el criterio de selección de la población en estudio se basa en la presencia (casos) o ausencia (controles) del evento en estudio y en que es el investigador quien fija el número de eventos a estudiar, así como el número de sujetos sin evento (controles) que se incluirán como población de comparación o referencia. De esta manera la población en estudio queda compuesta por un grupo de sujetos con el evento en estudio (casos) y un grupo de sujetos sin el evento (controles), posteriormente estos grupos se comparan en términos de la exposición que tuvieron al factor causal en estudio. A diferencia de los estudios de cohorte en los que se iguala la proporción de sujetos expuestos y no expuestos en la población de estudio, en este diseño se tiende a igualar la población en estudio en términos de los sujetos con y sin el evento en estudio.

En general, este tipo de estudios se lleva a cabo utilizando sistemas de registro que permiten identificar fácilmente a los sujetos que desarrollaron el evento en estudio (casos). Los sistemas de registro tradicionalmente utilizados incluyen centros hospitalarios o registros con base poblacional, como son los de neoplasias o malformaciones congénitas. El común denominador en este tipo de estudios es la utilización de un sistema que permite concentrar información sobre la población que presenta el evento en tiempos relativamente cortos y, en general, sin la necesidad de invertir cuantiosos recursos económicos, como los que se podrían requerir para concentrar el mismo número de eventos en el contexto de un estudio de cohorte. Mediante un mecanismo de selección, independiente al utilizado para los casos, se selecciona como grupo de contraste una serie de sujetos que no han desarrollado el evento en cuestión. La comparación directa de sujetos con (casos) y sin (controles) el evento, con relación al antecedente de exposición se utiliza con frecuencia para establecer asociaciones entre la exposición y el evento en estudio. Sin embargo, a pesar de que frecuentemente se utiliza esta comparación de manera automática para establecer asociaciones causales entre la exposición y el evento, es muy importante recalcar que para que ésta pueda considerarse como válida, se requiere del cumplimiento de ciertas condiciones sobre el origen de los casos y los controles. Entre otras cosas se requiere, por ejemplo, que los casos y controles tengan su origen en la misma base poblacional, que los controles representen de manera adecuada la población de donde provienen los casos y que cumplan con la condición de que si desarrollaran el evento en estudio, hubieran sido reclutados en la población de estudio como casos. Es claro que a menos de que el estudio se desarrolle al interior de una cohorte bien definida no será posible verificar el cumplimiento de las condiciones anteriormente mencionadas; sin embargo, el cumplimiento teórico de las mismas se podrá analizar en la medida que pensemos en los estudios de casos y controles como una alternativa para estudiar una cohorte imaginaria, la cual es posible delinear en tiempo y espacio, pero que es estudiada por medio de la selección de una muestra representativa de los eventos (casos) y de los individuos que no desarrollan el evento de interés (controles). El supuesto del origen común de los casos y controles se cumple si ambos se originan de la misma cohorte y representan tanto a los eventos como a la población en riesgo que no desarrolló el evento.

Los estudios de casos y controles son frecuentemente realizados de manera retrospectiva (figura 4), por lo que no tienen una relación causal perfecta, ya que el evento se evalúa antes que la causa y no siempre se puede inferir que la causa antecedió al evento. La naturaleza retrospectiva de los estudios de casos y controles hace que este tipo de estudios sea particularmente vulnerable a la introducción de errores en los procesos de selección o de recolección de la información. Por esta razón, este tipo de estudios se ha considerado tradicionalmente con un puntaje bajo en la escala de causalidad. Sin embargo, en ciertas ocasiones también es posible realizar este tipo de estudio de manera prospectiva y en ese contexto este tipo de estudios tiene mayor peso en la escala de causalidad. Las principales ventajas y desventajas de este tipo de estudios se describen en el cuadro IV.

\section{Est udios transversales}

Finalmente, la población en estudio puede ser seleccionada de manera aleatoria sin considerar la exposición o el evento como criterios de selección. Este tipo de estudio se ha denominado como de encuesta o transversal en los diferentes textos, ${ }^{1-4}$ y se distingue porque se indaga sobre la presencia de la exposición y la ocurrencia del evento una vez conformada la población en estudio, y porque sólo se hace una medición en el tiempo en cada sujeto de estudio (figura 5). El número de eventos así como la proporción de sujetos con la exposición están determinados por la frecuencia con que ocurren éstos en la población elegible y, por lo tanto, quedan fuera del control del investigador. Esto último contrasta con los estudios de cohorte o de casos y controles en los que el investigador puede fijar con anterioridad, ya sea la proporción de expuestos 


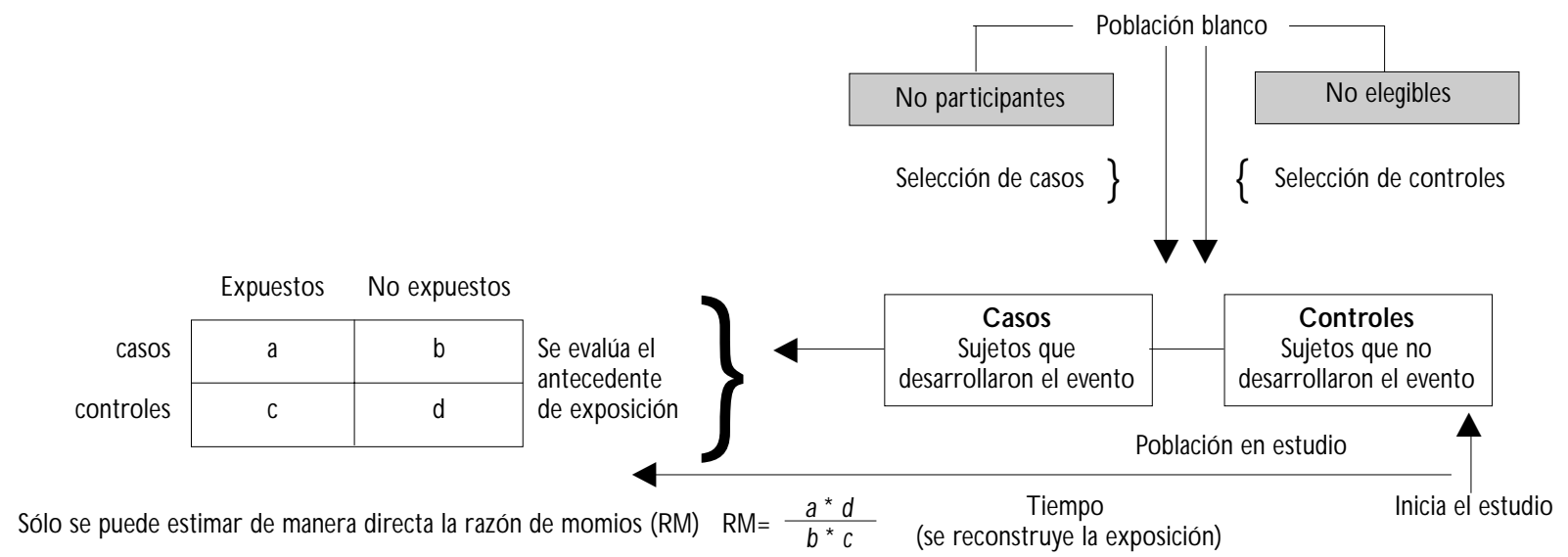

Figura 4. Estudios de CASOS Y CONTROLES

(estudio de cohortes) o la prevalencia del evento en la población en estudio (estudio de casos y controles). Los estudios transversales se caracterizan porque sólo se hace una medición en el tiempo en cada sujeto de estudio.

Este tipo de estrategia comparte muchas de las limitaciones de los estudios de casos y controles (cuadro $\mathrm{V}$ ), son retrospectivos y se basan en el estudio de casos prevalentes, los que en general representan a los sujetos con periodos de mayor sobrevida o duración de la enfermedad (evento). Cualquier factor que esté relacionado con la duración del evento y la exposición puede ser una fuente de error en este tipo de estudios. Por lo anterior, los estudios de encuesta tienen

\section{Cuadro IV \\ VentajAS Y DESVENTAJAS DE LOS ESTUdios DE CASOS Y CONTROLES}

Ventajas

- Eficientes para el estudio de enfermedades raras

- Eficientes para estudiar enfermedades con periodos de latencia 0 inducción prolongados

- Se pueden estudiar varias exposiciones simultáneamente

- En comparación con los estudios de cohorte son menos costosos y se pueden realizar en menor tiempo

Desventajas

- No se pueden estimar de manera directa medidas de incidencia 0 prevalencia

- Susceptibles a sesgos de selección

- Se puede presentar causalidad reversa

- Problemas para definir población fuente de los casos

- Problemas para medir adecuadamente exposición una escala baja en términos de causalidad y deben ser interpretados con mucha cautela. Sin embargo, son estudios útiles para la planeación de los servicios de salud y para caracterizar el estado de salud de la población en un punto en el tiempo.

\section{Estudios ecológicos o de conglomerado}

En general, en los diferentes tipos de estudios epidemiológicos que hemos mencionado, la unidad de análisis es el sujeto que compone la población en estudio, y es en éstos en los que se mide la exposición y se registra la ocurrencia del evento en el estudio. Sin embargo, en ocasiones la unidad de análisis puede no ser el individuo, sino un conjunto o conglomerado de individuos miembros de la población en estudio. Los conglomerados pueden estar constituidos por grupos poblacionales, comunidades, regiones, o países. La característica principal de este tipo de estudios es que se cuenta con información sobre la exposición o el evento para el conglomerado en su totalidad, desconociéndose la información a nivel individual para cada uno de los miembros del conglomerado. En este tipo de estudios es común asignar la misma exposición (exposición promedio) a todo el conglomerado, ignorando o no considerando la variación individual de cada miembro del conglomerado. Lo mismo sucede con la medición del evento; dado que sólo se cuenta con el número de eventos registrados para el conglomerado, no podemos discernir sobre los eventos que se presentaron en los sujetos expuestos de los que ocurrieron en los no expuestos, al interior de cada conglomerado, por lo que es frecuente atribuir la totalidad de eventos -sin una veri- 


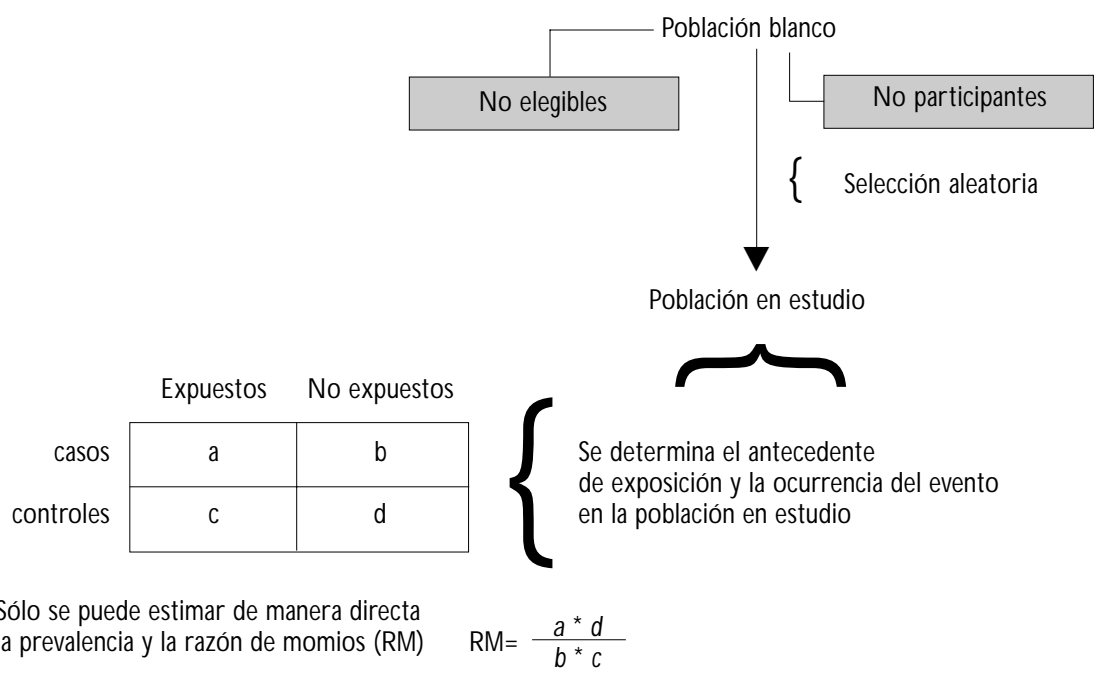

Figura 5. Estudio transversal o de encuesta

ficación real-a la exposición promedio que se registró al interior del conglomerado.

Los estudios de conglomerados (ecológicos) (cuadro VI) permiten estudiar grandes grupos poblacionales en poco tiempo y con un costo relativamente muy bajo, ya que en general utilizan estadísticas existentes recolectadas con otros fines. Sin embargo, dado que tienen el puntaje más bajo en la escala de causalidad deben ser considerados únicamente para sugerir hipótesis, que tendrán necesariamente que ser verificadas mediante otros estudios más rigurosos. Los principales problemas de este tipo de estudios son que se ignora la variabilidad individual de los integrantes de los conglomerados y que no es posible corregir por diferencias en otras variables que pudieran estar también asociadas con la exposición y el evento en estudio.

\section{Conclusiones}

Hemos revisado brevemente las principales estrategias de muestreo o diseños de investigación utilizados en los estudios epidemiológicos. Sin duda el ensayo aleatorizado es la estrategia que se reconoce como más poderosa para establecer relaciones causa efecto. Sin embargo, frecuentemente no es posible utilizar este tipo de diseño en estudios epidemiológicos, en particular cuando evaluamos los efectos de la exposición a sustancias tóxicas, que pueden ser de uso común pero que su aplicación deliberada a un grupo experimental sería inaceptable, en estas circunstancias tendremos que

\section{Cuadro V \\ Ventajas y DesVentajas de los estudios \\ TRANSVERSALES}

Ventajas

- Eficientes para estudiar la prevalencia de enfermedades en la población

- Se pueden estudiar varias exposiciones

- Son poco costosos y se pueden realizar en poco tiempo

- Se puede estimar la prevalencia del evento

Desventajas

- Problemas para definir y medir exposición

- Sesgos de selección

- Sesgos por casos prevalentes

- La relación causa efecto no siempre es verificable

- Sobrerrepresentación de enfermos con tiempos prolongados de sobrevida o con manifestaciones con mejor curso clínico

- Se puede presentar causalidad débil

basarnos en diseños de tipo observacional y realizar estudios en poblaciones que han estado expuestas por alguna circunstancia, ya sea de tipo ocupacional, ambiental o accidental.

Un ejemplo de la situación antes mencionada es la exposición al DDT. Pese a que se consideraría inaceptable la exposición intencional (experimental) de un grupo poblacional a este insecticida con el único fin de evaluar efectos tóxicos, un gran número de personas se expone frecuentemente a esta sustancia, ya sea por actividades ocupacionales o por vivir en regio- 


\section{Cuadro VI VentajAs y DESVENTAJAS DE LOS ESTUdios ECOLÓgICOS}

Ventajas

- Se pueden estudiar grandes grupos poblacionales

- Relativamente fáciles de realizar

- Aumenta el poder estadístico

- Aumenta la variabilidad en exposición

- Se puede utilizar información de estadísticas vitales

Desventajas

- No se tiene información del individuo por lo que no se puede ajustar por diferencias a nivel individual (no se puede saber quién sí está expuesto o quién sí desarrolló el evento de interés)

- No se tiene información sobre factores de confusión y no se puede coregir por éstos

nes palúdicas donde el DDT se utiliza como un método de control del paludismo.

A lo largo de los últimos años se ha desarrollado un número importante de estudios epidemiológicos cuyo objetivo principal ha sido el de evaluar si la exposición al DDT en poblaciones humanas tiene efectos sobre la salud, en particular se han evaluado los efectos reproductivos del DDT y su posible asociación con un incremento en el riesgo de cáncer. Mencionaremos algunos de ellos con el fin de ilustrar la utilización de los diferentes diseños epidemiológicos descritos anteriormente en un problema de actualidad en el campo de la salud pública.

Cocco y colaboradores ${ }^{6}$ realizaron un estudio de cohorte retrospectivo con el fin de estudiar la asociación entre exposición laboral al DDT y riesgo de desarrollar cáncer. Para este estudio se definió como población expuesta a los sujetos que participaron en el programa de control del paludismo en Sardenia, Italia. Los investigadores obtuvieron información sobre el estado de salud y la causa de muerte de los trabajadores que participaron en las actividades de control. En este grupo se registraron 1043 muertes, la distribución y frecuencia de causas de muerte observada se compararon con las que se registraron para la población italiana del mismo sexo y edad.

Hunter y colaboradores ${ }^{7}$ estudiaron la asociación entre los niveles séricos de DDE (indicador de exposición a DDT) y la incidencia de cáncer mamario. Este grupo de investigadores realizó un estudio de casos y controles prospectivo en una cohorte bien definida de enfermeras norteamericanas iniciado en 1986. Para el reporte antes mencionado se incluyeron en la población en estudio 240 casos de cáncer mamario que se registraron en la cohorte de enfermeras entre 1990 y 1992. Los niveles de DDE en suero se compararon con los observados en un grupo de participantes de la misma cohorte y del mismo grupo de edad, pero que no desarrollaron cáncer mamario. Los niveles de DDE se determinaron en muestras de suero que habían sido recolectadas en años previos al diagnóstico de cáncer mamario.

Romieu y colaboradores ${ }^{8}$ realizaron un estudio de casos y controles para estudiar la asociación entre la exposición ambiental a DTT y el riesgo de desarrollar cáncer mamario en mujeres residentes de la ciudad de México. En este trabajo, los investigadores estudiaron una serie de 120 casos incidentes de cáncer mamario reclutados en unidades médicas del Instituto Mexicano del Seguro Social, el Instituto de Seguridad y Servicios Sociales de los Trabajadores del Estado y la Secretaría de Salud, y compararon de manera concurrente los niveles de exposición a DDT, con los observados en una serie de 123 controles que se reclutaron mediante un muestreo aleatorio de mujeres de la ciudad de México.

Wang y colaboradores, ${ }^{9}$ realizaron un estudio multinacional de conglomerados para estudiar la asociación entre exposición al DDT y cáncer. En este estudio los autores recuperaron muestras de cerumen de 3800 sujetos entre 35 y 54 años de edad de diferentes poblaciones (35 países) y determinaron las concentraciones promedio de exposición a DDT en las muestras de cerumen. La concentración promedio de DDT fue utilizada para caracterizar la exposición promedio de cada país al DDT y posteriormente correlacionaron esta exposición promedio de DDT con la tasa de mortalidad por cáncer de los países incluidos en el estudio.

Finalmente, Ayotte y colaboradores* estudiaron la asociación entre los niveles plasmáticos de DDT y parámetros reproductivos determinados mediante una espermatobioscopía. Para fines de este estudio los investigadores reclutaron voluntarios de una de las zonas palúdicas de México y caracterizaron de manera transversal la asociación entre los niveles séricos de DDT y el número de espermatozoides, así como de otros parámetros funcionales. En este estudio también se evaluó de manera transversal la asociación del DDT en plasma con diferentes proteínas transportadoras de hormonas.

Los diferentes métodos epidemiológicos descritos en este trabajo representan los estudios más utilizados. La aplicación de cada método a diferentes problemas

* Ayotte P, Hernández-Avila M, Farías P, Villanueva CA, Giroux S, Dewailly E. Male reproductive function and endocrine profile in Mexican peasants exposed to $p, p^{\prime}$-DDEm. 
de salud requiere de creatividad y de conocimiento de las limitaciones de cada uno así como de los métodos de análisis que se han desarrollado de manera específica para cada aplicación. En los capítulos subsecuentes se presentará con mayor detalle cada uno de estos diseños.

\section{Referencias}

1.W alker AM. $O$ bservation and inference.An introduction to the methods of epidemiology. Chestnut Hill, MA: Epidemiology Resources Inc, 1991 2. Kelsey JL, Thompson W D, Evans AS. Methods in observational epidemiology. N ueva York: 0 xford University Press, 1986.
3. Kleinbaum DG, Kupper LL, Morgenstern H. Epidemiologic research. Principles and quantitative methods. Belmont, CA: Lifetime Learning Publications, 1982.

4. Rothman KJ, Greenland S. Modern epidemiology. 2a. edition. East W ashington Square, PA: Lippincott-Raven Publishers, 1998.

5. Miettinnen $0 \mathrm{~S}$. Theoretical epidemiology. Principles of occurrence research medicine. Nueva York: A W iley Medical Publication, 1985.

6. Cocco P, Blair A, Congia P, Saba G, EccaAR, Palmas C. Long-term health effects of the occupational exposure to DDT. A preliminary report. Ann NY A cad Sci 1997:837:246-256.

7. Hunter DJ. Hankinson SE. Laden F. Colditz GA. Manson JE.W illett W C et al. Plasmaorganochlorine levels and the risk of breast cancer [see comments]. N Engl J Med 1997;337(18):1253-1258.

8. Romieu I, Hernández M, Lazcano E, W eber JP, D ewali E. Breast cancer, lactation history and serum organochlorines. Am J Epidemiol 2000. En prensa.

9.W ang X Q.G ao PY. Lin YZ. Chen CM. Studies on hexachlorocyclohexane and DDT contents in human cerumen and their relationships to cancer mortality. Biomed Environ Sci 1988;1(2):138-151. 